\title{
Binocular summation of chromatic changes as measured by visual reaction time
}

\author{
J. R. JIMÉNEZ, J. M. MEDINA, L. JIMÉNEZ DEL BARCO, and J. A. DÍAZ \\ University of Granada, Granada, Spain
}

\begin{abstract}
We determined visual reaction times to monocular and binocular changes in the luminance of isochromatic stimuli and to monocular and binocular changes in the color of isoluminant stimuli. Two isoluminant color changes were tested: chromatic variations along the red-green axis of Boynton's (1986) two-stage color vision model and chromatic variations along the yellow-blue axis of the same model. The results indicate a greater degree of binocular summation for luminance change than for color change. This result was largely independent of the motor component of reaction time.
\end{abstract}

Visual patterns can be recognized and classified on the basis of either color-contrast-sensitive or luminancecontrast-sensitive mechanisms (Livingstone \& Hubel, 1988), and many attempts have been made to identify the relative contributions of both mechanisms to stereopsis. Much of this work was generated by the claim of Lu and Fender (1972) that color contrasts alone cannot support stereopsis; that is, one cannot perceive depth if the leftand right-eye stimulus patterns are defined by isoluminant color contrasts. Subsequent work (de Weert \& Sazda, 1983; Jiménez, Rubiño, Hita, \& Jiménez del Barco, 1997; Kingdom \& Simmons, 1996; Scharff \& Geisler, 1992; Simmons \& Kingdom, 1994, 1997), however, supports the existence of an independent color-contrast mechanism that is capable of stereopsis.

Because stereopsis arises from a comparison and fusion of the left- and right-eye images over a range of disparities, it would be interesting to determine whether color-contrast mechanisms would be capable of supporting other binocular phenomena, such as binocular summation. It has long been known that luminance-based detection thresholds are higher for monocular presentations than for binocular presentations, indicating the presence of binocular summation, and there have been a number of studies of binocular summation for suprathreshold stimuli (see Blake \& Fox, 1973; Blake, Sloane, \& Fox, 1981 , for reviews). The present study uses a visual reaction time (VRT) task to test for the presence of binocular summation when the stimulus pattern is defined by isoluminant color contrasts.

The first studies (Haines, 1977; Ueno, 1977) of binocular summation using a reaction time measure for de-

The authors thank David Simmons for reviewing the paper. David Nesbitt translated the original Spanish text into English, and Karen Shashok checked the English text for readability. We are also grateful to the anonymous referees for improving the content and clarity of the manuscript. Correspondence should be addressed to J. R. Jiménez, Departamento de Óptica, Facultad de Ciencias, Universidad de Granada, 18071 Granada, Spain (e-mail: jrjimene@ugr.es). tecting a change in chromaticity found evidence of binocular summation, but the stimuli contained both achromatic and chromatic variations. Later, Trick and Guth (1980) found binocular summation with dichoptically presented monochromatic stimuli of different wavelengths. This effect was more pronounced for saturated spectral hues (reds and violets) than for less saturated hues (yellow). Differences in summation varied by around $10 \%-15 \%$ between 650 and $575 \mathrm{~nm}$. Recently, Simmons and Kingdom (1998) studied binocular summation for compound stimuli (isoluminant red-green, isochromatic yellow-blue, or a combination of the two), determining binocular and monocular contrast-detection thresholds. Evidence was found for facilitation above probability summation within chromatic-contrast mechanisms.

In the present study we used VRT to determine the degree of binocular summation for suprathreshold stimuli under three conditions of observation (right and left monocular, and binocular) and two experimental conditions (isochromatic luminance variation and isoluminant chromatic variation). To determine the degree of binocular summation for chromatic stimuli, we used the chromatic differences at isoluminance according to red-green (S-constant cone axis) and S directions (L \& M-constant cone axis) of Boynton's two-stage color-vision model. This approach is more informative since stimulus selection is based on a color-perception model, taking into account the first stages in color processing (Boynton, 1986; de Valois \& de Valois, 1993; Guth, 1991).

\section{METHOD}

\begin{abstract}
Apparatus
The stimuli were presented on a CRT color monitor connected to a microcomputer equipped with an 8-bit graphics card. The monitor was adjusted to a resolution of 1,024 points per 768 lines, with vertical frequency scanning of $74 \mathrm{~Hz}$. Chromaticity and luminance of the stimuli were controlled by periodic calibrations using a SpectraScan PR-704 PhotoResearch spectroradiometer. To produce stimuli with specific CIE-1931 coordinates and luminance, we used a modification of the calibration method of Post and Calhoun (1989) proposed
\end{abstract}


Table 1

Values (in Trolands) for the Stimuli Distributed Along L \& M (S Constant) and S (L \& M Constant) Directions at Isoluminance, According to Boynton's (1986) Model

\begin{tabular}{cc}
\hline Stimuli Selected Along L \& M & Stimuli Selected Along S \\
\hline-2.99 & 83.98 \\
-5.71 & 71.34 \\
-8.32 & 58.99 \\
-11.00 & 46.38 \\
-13.61 & 34.26 \\
-16.26 & 21.70 \\
2.34 & 108.86 \\
5.08 & 121.06 \\
7.62 & 133.79 \\
10.31 & 145.70 \\
13.04 & 158.67 \\
15.55 & 171.41 \\
\hline
\end{tabular}

Note-All L, M, and S values are given according to the luminance of the reference stimulus.

by Jiménez del Barco, Díaz, Jiménez, and Rubiño (1995). Observers were seated $70 \mathrm{~cm}$ from the CRT in a dark room, and a chinrest was used for head stabilization.

\section{Stimuli}

Luminance stimuli. We used 12 stimuli, with chromaticity coordinates equal to those of the equal-energy illuminant $(x=.333$, $y=.333$ ), with luminance values $(\mathrm{L}+\mathrm{M})$ between 3 and $27 \mathrm{~cd} / \mathrm{m}^{2}$, at intervals of $2 \mathrm{~cd} / \mathrm{m}^{2}$. The reference stimulus was an achromatic stimulus of $15 \mathrm{~cd} / \mathrm{m}^{2}$ (the chromaticity coordinates of which were also identical to the equal-energy illuminant).

Chromatic stimuli. For each observer and observational condition (monocular and binocular), a minimum-flicker photometric procedure at a frequency of $12.3 \mathrm{~Hz}$ was used to match the luminance of each stimulus with the reference stimulus. To generate the variations along the constant $\mathrm{L} \& \mathrm{M}$ cone axis, we chose 12 stimuli, distributed symmetrically around the reference stimulus in the chromaticity diagram CIE-1931 $(x, y)$, according to a red-green direction originating at point $(x=1.0, y=0)$ (Boynton, 1986). To generate the $\mathrm{S}$ variations, we chose 12 stimuli distributed symmetrically around the reference according to a tritan confusion line originating at point $(x=.175, y=0)$ (Boynton, 1986). Table 1 shows the values of the stimuli obtained according to Boynton's model, and Figure 1 presents the stimuli plotted in the CIE-1931 chromatic diagram with the luminance level of the reference stimulus.

All stimuli could be clearly discriminated with respect to the reference stimulus; therefore VRT was determined under suprathreshold conditions, as in the study of Blake, Martens, and Di Gianfilippo (1980). Several color-difference formulas (Brainard, 1995) were used to confirm that the color stimuli were clearly discriminated. In this part of the experiment, the VRT was determined by chromatic changes at isoluminance, the observer detecting only hue changes, and the reference stimulus having the same luminance as those that were to be detected (Bowen, 1981; Nissen \& Pokorny, 1977; Smith, Bowen, \& Pokorny, 1984).

All stimuli were uniform circular patches $1.5^{\circ}$ in diameter and were presented on a dark background. They were observed foveally with the natural pupil (Blake et al., 1980; Haines, 1977; Westendorf \& Blake, 1988) under three conditions: right and left monocular (in this case one eye was covered with a black patch) and binocular.

\section{Procedure}

VRTs to isochromatic stimuli varying only in luminance were obtained first, followed by VRTs to isoluminant stimuli varying in hue along the red-green axis and then along the yellow-blue axis. The order of presentation of the observation conditions (binocular, monocular right, monocular left) was randomly determined within each experimental session. At the beginning of a session the subject was allowed 3 min to adapt to darkness and then 3 additional min to adapt to the reference stimulus. The beginning of a trial was signaled by a tone. After a random delay (3-7 sec, uniform sampling

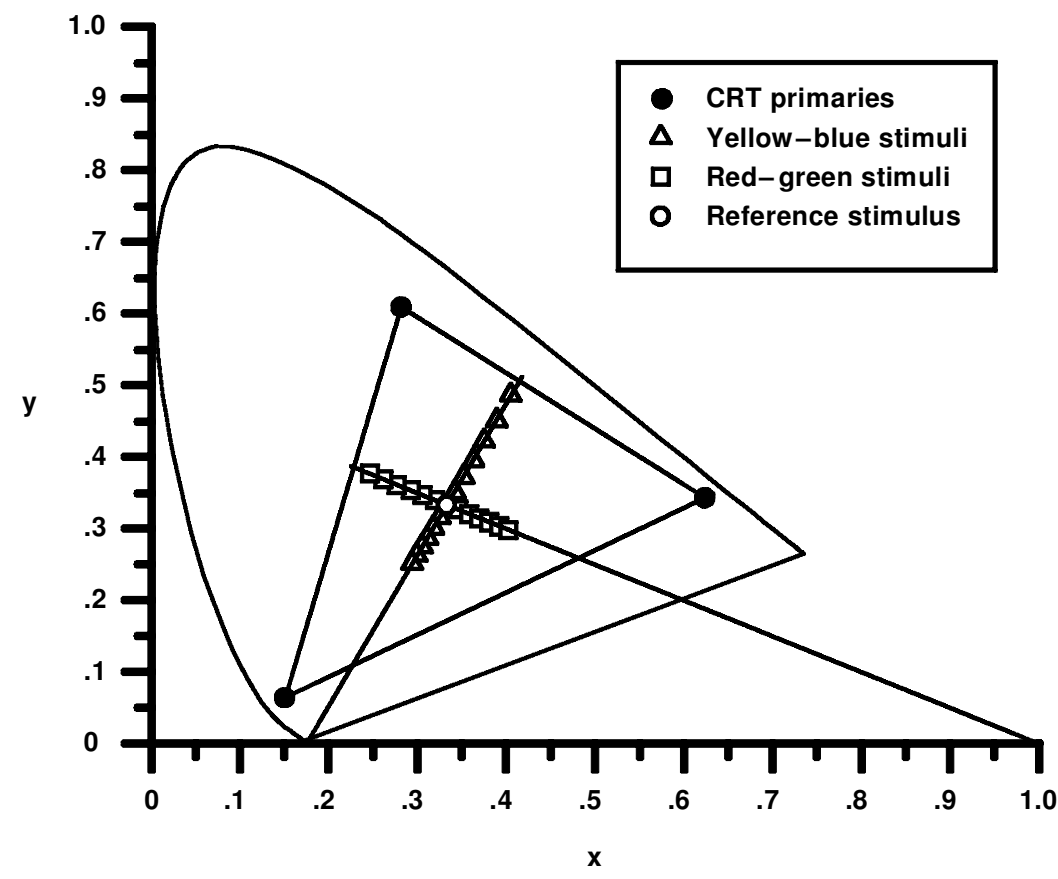

Figure 1. Representation in the CIE-1931 chromatic diagram of the chromatic stimuli at isoluminance used in the experiments. Level of reference luminance: $15 \mathrm{~cd} / \mathrm{m}^{2}$. 
distribution), the reference stimulus was changed to the test stimulus with the change synchronized to the beginning of the refresh cycle. The test stimulus that replaced the reference stimulus remained on until the subject responded by pressing the button on the mouse connected to the microcomputer to indicate that a stimulus change had occurred. Immediately following a response, the test stimulus was replaced by the reference stimulus.

Note that in the isochromatic luminance variation condition, changing from the reference stimulus to a test stimulus is equivalent to changing the luminance of an isochromatic stimulus. In the isoluminant chromatic variation conditions, a change from the reference stimulus to a test stimulus results in a hue change, but not a luminance change in the stimulus. Each test stimulus was randomly presented a total of eight times during a session. VRT was measured as the time between the change in the stimulus and the pressing of the mouse button. The computer clock was programmed to provide 1-msec timing (Sheppard, 1987).

In each session, we eliminated the first four reaction times; in addition, those below $180 \mathrm{msec}$ were discarded as false alarms, and those exceeding $950 \mathrm{msec}$ were excluded as misses. We performed a number of sessions until no fewer than 80 VRTs had been collected for each test stimulus under each experimental condition. The VRT value for each change in stimulus was taken as the arithmetic mean of the distribution. For each subject's data and for each experimental condition, signif icant differences were determined from an independent measures $3 \times 12$ analysis of variance (ANOVA) with observation (binocular, left, and right eye) and experimental conditions (luminance or L \& M or S variations) as the factors, as well as from a multiple-comparison test (the Scheffé method). For all statistical analyses, $\alpha=.05$ was accepted as the minimum level of significance (Martin \& Luna del Castillo, 1990).

\section{Observers}

The subjects were 3 of the 4 authors (J.A., J.M., and J.R., 32, 27, and 34 years old, respectively), a number similar to that in other studies of binocular summation (Simmons \& Kingdom, 1998;
Ueno, 1977; Westendorf \& Blake, 1988). All the observers who participated in the experiments had normal color vision (according to the Ishihara test, Pickford-Nichol son anomaloscopy and Dichotomique Farnsworth 15D test) and normal stereopsis (according to stereo-fly tests). To minimize the effects of learning and to establish the magnitude of the VRT for each experimental condition, all the observers were given training sessions that lasted roughly 3-5 months. The VRT data were subjected to independent measures three-way ANOVAs for each observer's data and for each experimental condition in a $2 \times 3 \times 12$ factorial design with the second-to-the-last and the last set of VRT data, observation (binocular, left, and right eye), and experimental conditions (luminance or $\mathrm{L}$ $\& \mathrm{M}$ or $\mathrm{S}$ variations) used as the main factors. This process was considered finalized when no statistical differences were found between the next-to-the-last and the last set of VRT. All VRT measurements prior to the last dataset were discarded from the subsequent analysis.

\section{RESULTS}

\section{Binocular Summation for Luminance and Chromaticity Variations}

Figure 2 shows mean overall VRT for Observers J.A., J.M., and J.R. These values were determined, for each observational condition, as the arithmetic mean for all VRT means according to the different experimental conditions (vertical bars include $+1 S E M$ ). For example, for each observer the right mean value of VRT for the luminance stimuli $(\mathrm{L}+\mathrm{M})$ was calculated by averaging the 12 mean VRT obtained, as in the study by Blake et al. (1980) on binocular summation and VRT for luminance changes.

It is notable that we detected a gradation in Figure 2 for VRT data under all three experimental conditions. The mean VRT proved shorter for luminance than for

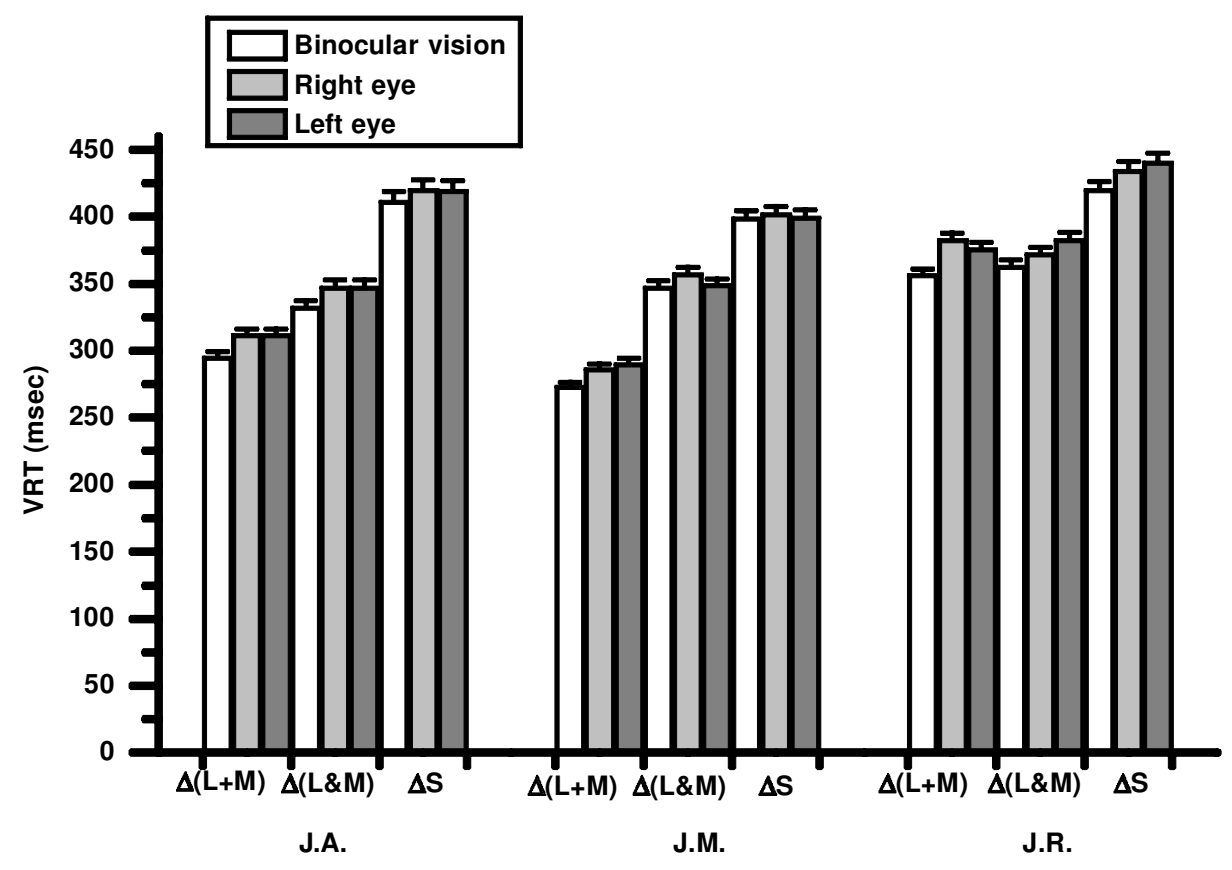

Figure 2. Mean overall visual reaction time values (msec) for each observational condition (binocular and both monocular) for the different groups of experimental conditions: luminance $\Delta(\mathbf{L}+\mathbf{M})$ variations, $\Delta(\mathbf{L} \& \mathrm{M})$, and $\Delta S$ at isoluminance. Data are presented separately for Observers J.A., J.M., and J.R. Error bars include +1 SEM. 
$\mathrm{L} \& \mathrm{M}$ variations, and both in turn were shorter than the mean VRT for $\mathrm{S}$ variations. This trend was confirmed under all observational conditions except in the case of Observer J.R. for the right eye, for which the mean VRT for the luminance signal was greater than that for the red-green signal. These results are consistent with those found by Jiménez del Barco, Martinez, Jiménez, Rubiño, and Hita (1992) and Baker and Mollon (1995). It bears emphasizing that this tendency was also appreciable in binocular vision for each of the 3 observers, for whom the binocular mean VRT was shorter for luminance changes than was the binocular mean VRT for L \& M changes, which in turn were shorter than the binocular mean VRT for $\mathrm{S}$ variations.

Luminance variations. An initial analysis of the results for the luminance signal for all 3 observers confirmed significant differences in the main effect of observational conditions [binocular, right and left eye: $F(2,2469)=23.87, p<.001$ for J.A., $F(2,2443)=32.07$, $p<.001$ for J.M., and $F(2,2367)=45.42, p<.001$ for J.R.]. The subsequent Scheffé test indicated significant differences in the binocular VRT with respect to the right (all subjects, $p<.001$ ) as well as the left eye (all subjects, $p<.001$ ). This test also indicated for each observer that the VRT for the right and left eye did not differ significantly ( $p=.78$ for J.A., $p=.32$ for J.M., and $p=.30$ for J.R.). VRT was also found to be shorter for binocular than for monocular viewing, as reported in other studies (Blake et al., 1980; Gilliland \& Haines, 1975; Haines, 1977; Minucci \& Connors, 1964; Ueno, 1977; Westendorf \& Blake, 1988).

Chromaticity changes: $\Delta(\mathbf{L} \& \mathbf{M})$ and $\Delta S$ variations at isoluminance. For Observers J.A. and J.R., ANOVA revealed significant differences in the main effect of observational conditions (binocular, left and right eye), for variations in both the red-green $[F(2,2232)=$ $11.89, p<.001$ for J.A. and $F(2,2601)=26.40, p<.001$ for J.R., respectively] and the yellow-blue signals $[F(2,2340)=9.78, p<.003$ for J.A. and $F(2,2464)=$ $18.04, p<.001$ for J.R.]. For both types of variation the Scheffé test indicated significant differences in binocular VRT with respect to the right eye $(p<.001$ for red-green and yellow-blue stimuli for J.A.; $p<.003$ for red-green and yellow-blue stimuli for J.R.) and the left eye $(p<.001$ for red-green and yellow-blue stimuli for J.A. and J.R.). In addition, there were no significant differences between the right and left eye ( $p=1.00$, red-green and yellow-blue stimuli) for J.A.; but for J.R. differences were found for red-green $(p<.002)$ but not for yellow-blue $(p=.36)$ stimuli. For both $\Delta(\mathrm{L} \& \mathrm{M})$ and $\Delta \mathrm{S}$ variations, binocular mean VRT was smaller than in monocular viewing. This verifies the existence of the summation phenomenon in J.A. and J.R. On the other hand, for Observer J.M., a significant difference was found between binocular and monocular viewing for the redgreen signal $[F(2,2523)=7.46, p<.002]$, but not for variations in the yellow-blue signal $[F(2,2448)=0.05$, $p=.95]$. The Scheffé test for variations in the red-green signal indicated differences between binocular viewing and the right eye $(p<.002)$ and between the right and left eyes $(p<.016)$ but not between binocular viewing and the left eye $(p=.77)$. For this observer, these results show a clearly reduced binocular summation.

\section{Estimation of the Summation Ratio}

When no monocular differences exist for normal observers, the traditional method used to evaluate the summation ratio, defined as the quotient between the average for the monocular and binocular VRTs (Blake et al., 1980; Lema \& Blake, 1977), assumes that equal ratios for reaction time correspond to equal strengths of binocular summation, without taking into account the difficulty of the task involved. However, this method is inadequate for determining binocular summation for the different experimental conditions we used (luminance and chromatic variations at isoluminance) due to the gradation in task difficulty shown in Figure 2. One possible way to estimate binocular facilitation, taking these points into consideration, would be to control the VRT ranges by subtracting the results of binocular trials from those obtained with monocular viewing, in a way similar to the expression used by Miller (1986) for intersensorial facilitation. However, this latter method expresses binocular facilitation in units (msec) and the results can be compared only for VRT experiments, thus limiting the possibility of generalizing the conclusions to those drawn with other types of experiments. We therefore preferred the following expression:

$$
\left(\frac{\left(\frac{\mathrm{VRT}_{\mathrm{R}}+\mathrm{VRT}_{\mathrm{L}}}{2}\right)-\mathrm{VRT}_{\mathrm{B}}}{\left(\frac{\mathrm{VRT}_{\mathrm{R}}+\mathrm{VRT}_{\mathrm{L}}}{2}\right)}\right) \times 100,
$$

where $\mathrm{VRT}_{\mathrm{R}}, \mathrm{VRT}_{\mathrm{L}}$, and $\mathrm{VRT}_{\mathrm{B}}$ refer to the VRT for the right eye, the left eye, and binocular viewing, respectively. This method expresses the improvement gained over monocular viewing as a percentage, while preserving the advantage of the two previous methods (since it has no units and takes into account the discrimination conditions for each type of experiment). When differences were detected with monocular viewing, as occurred for Observers J.M. and J.R. with variations in the red-green signal, binocular summation was determined with a conservative expression:

$$
\left(\frac{\min \left(\mathrm{VRT}_{\mathrm{R}}, \mathrm{VRT}_{\mathrm{L}}\right)-\mathrm{VRT}_{\mathrm{B}}}{\min \left(\mathrm{VRT}_{\mathrm{R}}, \mathrm{VRT}_{\mathrm{L}}\right)}\right) \times 100,
$$

where $\min \left(\mathrm{VRT}_{\mathrm{R}}, \mathrm{VRT}_{\mathrm{L}}\right)$ indicates the shorter of the two monocular reaction times. We thus calculated the binocular-summation ratio as the arithmetic mean of all binocular-summation ratios computed across all stimulus values for each type of experimental condition [luminance, $\Delta(\mathrm{L} \& \mathrm{M})$, and $\Delta \mathrm{S}]$, as has been done in other 
studies to calculate an overall average binocular summation ratio (Blake et al., 1980; Lema \& Blake, 1977; Simmons \& Kingdom, 1998).

The results for all observers (Figure 3, vertical bars include +1 SEM) showed clear binocular summation for luminance variations and reduced summation for chromaticity changes. Thus, for Observer J.A., the average difference for luminance was $5.26 \%$ between monocular and binocular VRT, whereas for chromatic variations this percentage was lower, falling to $4.24 \%$ and $3.40 \%$ for the red-green and $\mathrm{S}$ variations, respectively. The same trend was found in Observer J.R., for whom the average difference for luminance was $6.34 \%$, whereas the corresponding values for the red-green and yellow-blue signals were $1.82 \%$ and $3.69 \%$, respectively. For $\mathrm{Ob}-$ server J.M., this trend was even more obvious, the corresponding values being $5.47 \%$ for the change in luminance and $-0.47 \%$ and $0.28 \%$, respectively, for red-green and $\mathrm{S}$ variations. For this observer the ANOVA showed an absence of, rather than a reduction in, binocular summation for chromaticity changes.

In light of these results we investigated whether the motor component of reaction time exerted any influence. It should be taken into account that if we consider VRT as the sum of visual latency plus the motor component, then the latter could partially mask the degree of binocular summation, given that this is the main component of VRT and that it does not involve visual processing. We thus estimated the motor component from the data on luminance variations, assuming (as other researchers propose) that VRT can be expressed according to a Pièrontype law (Mansfield, 1973; Ueno, 1977):

$$
\mathrm{VRT}=\mathrm{VRT}_{\mathrm{o}}+\mathrm{b}\left(\mathrm{L}-\mathrm{L}_{\mathrm{o}}\right)^{-\mathrm{c}},
$$

where $\mathrm{VRT}_{\mathrm{o}}$ indicates the constant part of visual reaction time (asymptotic latency), which includes the motor component. In this equation $\mathrm{b}$ and $\mathrm{c}$ are constants, and the expression $b\left(L+L_{o}\right)^{-c}$ contains information on the VRT component that depends on luminance changes. We then determined the asymptotic latency for J.A., J.M., and J.R. For each observer, a one-way ANOVA on VRT for each observation condition showed no statistically significant differences between monocular and binocular viewing $[F(2,3)=0.57, p=.62$ for J.A.; $F(2,3)=0.15$, $p=.86$ for J.M., and $F(2,3)=1.80, p=.30$ for J.R.]. The asymptotic latency was then removed from each observational condition, both for those determined by luminance changes and for those corresponding to chromatic changes at isoluminance. Figure 4 illustrates the summation ratio resulting from deletion of the motor component (vertical bars include $+1 S E M$ ). Again, there was evidence for a clearcut order in the average binocular summation for the luminance variations $(52.47 \%$, $27.94 \%$, and $50.26 \%$ for Observers J.A., J.M., and J.R., respectively) compared with the chromatic variations at isoluminance (Observer J.A.: 22.59\% for L \& M variations and $8.98 \%$ for $\mathrm{S}$ variations; Observer J.R.: $14.96 \%$ for $\mathrm{L} \& \mathrm{M}$ variations and $15.48 \%$ for $\mathrm{S}$ variations; $\mathrm{Ob}-$ server J.M.: $-2.21 \%$ for $L \& M$ variations and $0.40 \%$ for $\mathrm{S}$ variations).

The gradation in binocular summation found for luminance and chromaticity variations not only confirmed the results found before the motor component was removed but also accentuated these differences, as indicated by the percentages cited earlier, and by Figure 4 . It should be mentioned that it is not possible to conclude definitively whether a similar trend occurs between chromatic red-green and yellow-blue signals, given

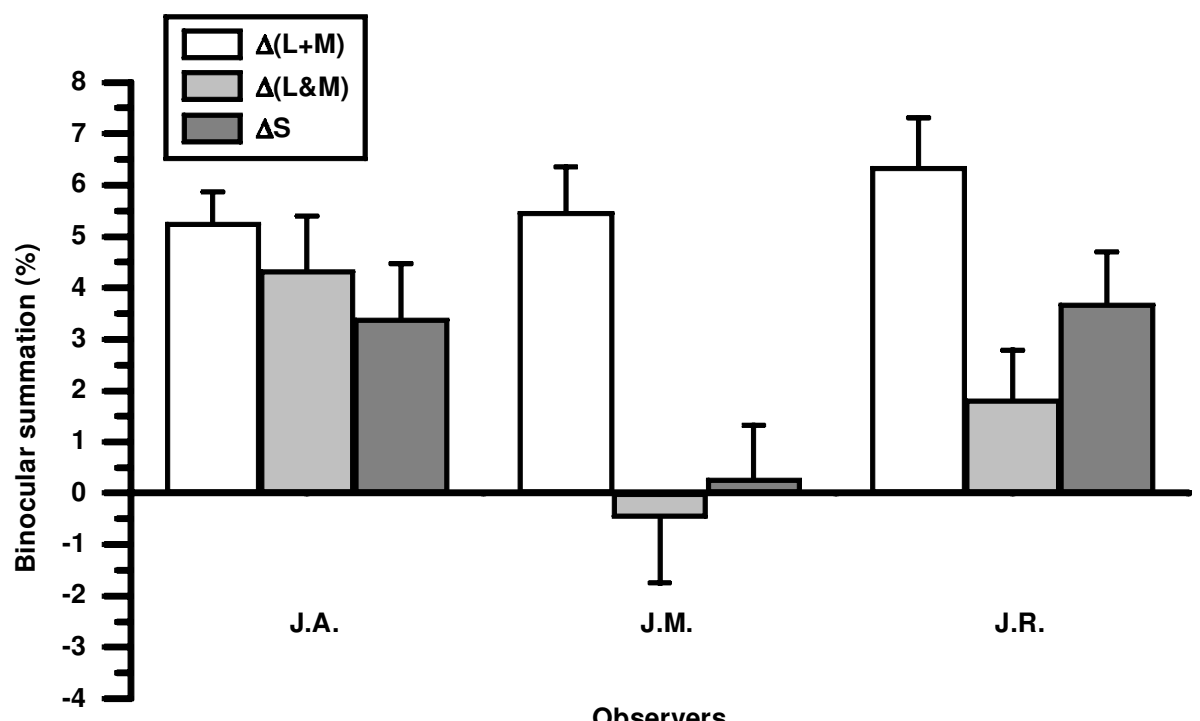

Figure 3. Binocular-summation ratio averaged across all experimental conditions: luminance $(\mathrm{L}+\mathrm{M})$ variations, $\Delta(\mathbf{L} \& \mathbf{M})$, and $\Delta \mathrm{S}$ at isoluminance. Data are presented separately for Observers J.A., J.M., and J.R. Error bars represent +1 SEM. 


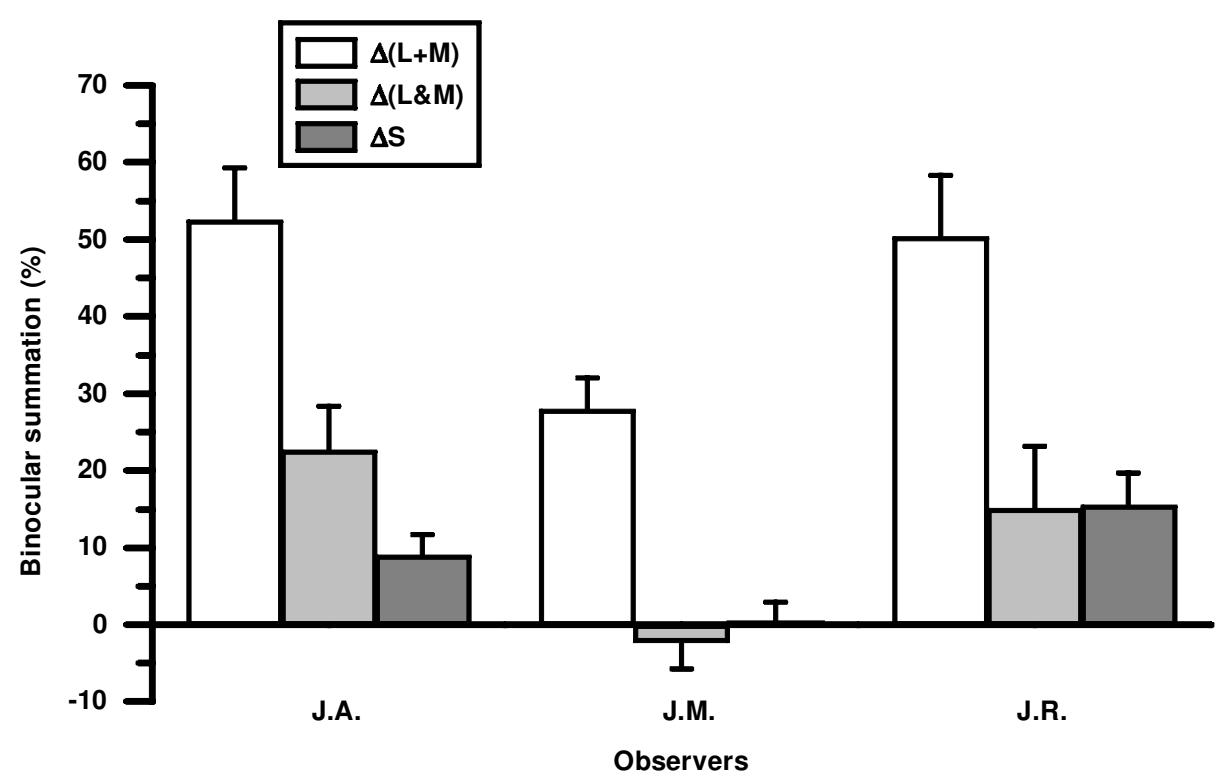

Figure 4. Binocular-summation ratio without considering asymptotic latency, averaged across all experimental conditions tested [luminance $(L+M)$ variations, $\Delta(L \& M)$, and $\Delta S$ at isoluminance]. Data are presented separately for Observers J.A., J.M., and J.R. Error bars represent +1 SEM.

that, in contrast to J.A., who did manifest such a trend, the binocular summation ratios for J.M. and J.R. were similar for both types of signals (Figure 4).

\section{DISCUSSION}

The reduction in binocular summation for chromatic variations in Observers J.A. and J.R., as well as the slight improvement for J.M. with respect to the yellow-blue signal $(0.40 \%)$, led us to consider previous studies that related the reduction in binocular summation to decreased stereopsis (Blake et al., 1980; Lema \& Blake, 1977; Levi, Harwerth, \& Manny, 1979; Rose, Blake, \& Halpern, 1988; Simmons \& Kingdom, 1998). In our study we confirmed that the degree of binocular summation was reduced for chromatic variations at isoluminance and surmised that it would be useful to study whether stereopsis at isoluminance was decreased in the observers who participated in our experiments. Accordingly, we analyzed the influence of chromatic information on the level of disparity detected by the observer. It should be borne in mind that disparity is the parameter that is processed to achieve stereoscopic depth perception. In Observer J.R., who had participated in previous experiments (Jiménez et al., 1997) that investigated stereopsis and color, we found, on determining the disparity range (maximum disparity due to displacement in the small square that generates stereopsis in the random-dot stereograms), that this range was higher for luminance variations than when the stereogram was generated with variations in chromaticity. This finding indicated that luminance variations were more effective than chromatic variations at isoluminance in facilitating stereo correspondence. The same tendency was evident in Observer J.A., who also took part in experiments on stereopsis and color (Jiménez et al., 1997) and who showed a more reduced disparity range for chromaticity variations than for luminance variations (the range was almost null for $\mathrm{S}$ variations). These findings are in agreement with the trend found for both observers in this experiment: reduced binocular summation for chromaticity variations relative to the effect brought about by luminance changes.

For J.M., who had not participated in previous experiments on stereopsis and color, we calculated the disparity range for RDS generated with variations in each of the three signals. The results showed a gradation in the disparity range for all three signals, with the disparity range for luminance variations being clearly greater (an average of $52^{\prime}$ ) than for chromaticity variations (an average of $26^{\prime}$ ). The results were analogous to those of J.R. and J.A.-namely, more reduced stereopsis for chromatic variations at isoluminance. In sum, for all $3 \mathrm{ob}-$ servers, we found a qualitative correlation between binocular summation in VRT and stereopsis, both effects being reduced in the presence of chromaticity variations.

It is worth noting that the relationship between binocular summation and stereopsis does not necessarily contradict the results of Simmons and Kingdom (1998), which showed evidence of a relationship in the opposite direction between the two binocular phenomena. Our chromatic stimuli were chosen along S and L \& M directions, maintaining the excitation level of the remaining chromatic channel constant for each case, thereby minimizing the possible interactions between the two 
chromatic mechanisms. This was not guaranteed in the work of Simmons and Kingdom, and this difference in procedure may therefore account for the greater degree of summation found for color as opposed to luminance. In this connection, both binocular phenomena (stereopsis and binocular summation) show performance decrements with chromatic signals at isoluminance. These trends suggest the lower effectiveness of chromatic processing with respect to luminance processing in certain aspects of binocular vision.

This evidence for the lower efficiency of chromatic variations in binocular phenomena is also consistent with the findings for monocular vision. It is known that the visual effectiveness of luminance and chromatic signals are not equal for the observer-that is, luminance variations are more efficient than chromatic ones in tasks involving stimulus discrimination, spatial vision, temporal aspects, and movement (de Valois \& de Valois, 1990; Geisler, 1995; Wandell, 1995; Yeh, Pokorny, \& Smith, 1993). In addition, the visual effectiveness of luminance signals is also confirmed by analysis of the ideal observer based on signal theory, which holds that less chromatic information at the level of the photopigments is available relative to luminance information (Geisler, 1995). Nonetheless, this latter fact did not completely account for the results concerning binocular summation for luminance with respect to chromaticity changes at isoluminance. The temporal response properties of chromatic mechanisms can be described as quasisustained (Ueno, 1992) and therefore as slow-action mechanisms. This behavior contrasts with the mechanism that processes luminance information, which is transitory (Ueno, 1992) and therefore fast acting. Thus, when the visual system processes chromatic information at isoluminance, binocular red-green and yellow-blue channels can be considered to be second-order mechanisms with regard to the greater efficiency of binocular luminance channels.

Our experimental data on VRT confirm the reduction in binocular summation for chromatic variations at isoluminance and show that chromaticity changes are processed less efficiently by the visual system than are luminance changes. This conclusion was strengthened when we analyzed data for VRT after removing the motor component. This information should be incorporated into models of visual processing that attempt to analyze the interaction between binocular information and color.

\section{REFERENCES}

Baker, M. R., \& Mollon, J. D. (1995). Using discriminative reaction times to estimate equal luminance. Perception, 24, 16.

Blake, R, \& Fox, R. (1973). The psychophysical inquiry into binocular summation. Perception \& Psychophysics, 14, 161-185.

Blake, R., Martens, W., \& Di Gianfilippo, A. (1980). Reaction time as a measure of binocular interaction in human vision. Investigative Ophthalmology\& Visual Science, 19, 930-941.

Blake, R., Sloane, M., \& Fox, R. (1981). Further developments in binocular summation. Perception \& Psychophysics, 30, 266-276.
Bowen, R. W. (1981). Latencies for chromatic and achromatic visual mechanism. Vision Research, 21, 1457-1466.

Boynton, R. M. (1986). A system of photometry and colorimetry based on cone excitations. Color Research \& Application, 11, 244252.

Brainard, D. (1995). Handbook of optics I (pp. 1-48). New York: McGraw-Hill.

dE VAlois, R. L., \& DE VAlois, K. K. (1990). Spatial vision (pp. 212238). Oxford: Oxford University Press.

DE VAlois, R. L., \& DE VALOIS, K. K. (1993). A multi-stage color model. Vision Research, 33, 1053-1065.

dE WeErT, C. M. M., \& SAZDA, K. J. (1983). New data concerning the contribution of color differences to stereopsis. In J. D. Mollon \& L. T. Sharpe (Eds.), Color vision: Physiologyand psychophysics (pp. 553562). London: Academic Press.

GeISLER, W. S. (1995). Discrimination information in natural radiance spectra. In X. Editor (Ed.), Vision models for target detection and recognition (pp. 117-131). London: World Scientific.

Gilliland, K., \& Haines, R. F. (1975). Binocular summation and peripheral visual response time. American Journal of Optometry \& Physiological Optics, 52, 834-839.

GutH, S. L. (1991). Model for color vision and light adaptation. Journal of the Optical Society of America, 8, 976-993.

HAINES, R. F. (1977). Visual response time to colored stimuli in peripheral retina: Evidence for binocular summation. American Journal of Optometry \& Physiological Optics, 54, 387-398.

JiméneZ, J. R., Rubiño M., HitA, E., \& JimÉnez del BARCo, L. (1997). Influence of the luminance and opponent chromatic channels on stereopsis with random-dot stereograms. Vision Research, 37, 591596.

Jiménez del Barco, L., Díaz, J. A., Jiménez, J. R, \& Rubiño, M. (1995). Considerations on the calibration of color displays assuming constant-channel chromaticity. Color Research \& Application, 20, 377-387.

Jiménez del Barco, L., Martinez, P., Jiménez J. R., Rubiño, M., \& HitA, E. (1992). Temporal response of opponent-chromatic channels to changes of chromaticity. Perception, 21, 79.

Kingdom, F. A. A., \& Simmons, D. R. (1996). Stereoacuity and color contrast. Vision Research, 36, 1311-1319.

LEMA, S. A., \& BlAKe, R. (1977). Binocular summation in normal and stereoblind humans. Vision Research, 17, 691-695.

Levi, D. M., Harwerth, R. S., \& Manny, R. E. (1979). Suprathreshold spatial frequency detection and binocular interaction in strabismic and anisometropic amblyopia. Investigative Ophthalmology \& Visual Science, 18, 714-725.

Livingstone, M. S., \& Hubel, D. H. (1988). Segregation of form, color, movement and depth: Anatomy, physiology and perception. Science, 240, 740-749.

Lu, C., \& Fender, D. H. (1972). The interaction of color and luminance in stereoscopic vision. Investigative Ophthalmology \& Visual Science, 11, 482-489.

Mansfield, R. J. W. (1973). Latency stimulus in human vision. Vision Research, 13, 2219-2234.

Martin, A., \& Luna del Castillo, J. (1990). Bioestadística para las ciencias de la salud. Madrid: Norma Editors. S. A.

Miller, J. (1986). Timecourse of coactivation in bimodal divided attention. Perception \& Psychophysics, 40, 331-343.

Minucci, P. K., \& Connors, M. M. (1964). Reaction time under three viewing conditions: Binocular, dominant eye, and nondominant eye. Journal of Experimental Psychology, 67, 268-275.

Nissen, M. J., \& Pokorny, J. (1977). Wavelength effects on simple reaction time. Perception \& Psychophysics, 22, 457-462.

Post, D. L., \& Calhoun, C. S. (1989). An evaluation of methods producing desired color on CRT monitors. Color Research \& Application, 14, 172-186.

Rose, D., Blake, R. \& Halpern, D. L. (1988). Disparity range for binocular summation. Investigative Ophthalmology \& Visual Science, 29, 283-290.

ScharfF, L. V., \& GeISLER, W. S. (1992). Stereopsis at isoluminance in the absence of chromatic aberrations. Journal of the Optical Society of America A, 9, 868-875. 
SHEPPARD, B. (1987, January). High performance software analysis on the IBM PC. Byte, pp. 157-164.

Simmons, D. R., \& Kingdom, F. A. A. (1994). Contrast thresholds for stereoscopic depth identification with isoluminant and isochromatic stimuli. Vision Research, 34, 2971-2982.

Simmons, D. R., \& Kingdom, F. A. A. (1997). On the independence of chromatic and achromatic stereopsis mechanisms. Vision Research, 37, 1271-1280.

Simmons, D. R., \& Kingdom, F. A. A. (1998). On the binocular summation of chromatic contrast. Vision Research, 38, 1063-1071.

Smith, V. C., Bowen, R. W., \& Pokorny, J. (1984). Threshold temporal integration of chromatic stimuli. Vision Research, 24, 653-660.

Trick, G. L., \& GUTH S. L. (1980). The effect of wavelength on binocular summation. Vision Research, 20, 975-980.
Ueno, T. (1977). Reaction time as a measure of temporal summation at suprathreshold levels. Vision Research, 17, 227-232.

UENO, T. (1992). Sustained and transient properties of chromatic and luminance systems. Vision Research, 32, 1055-1065.

WANDELL, B. A. (1995). Foundations of vision. Sunderland, MA: Sinauer.

Westendorf, D., \& Blake, R. (1988). Binocular reaction time data to contrast increments. Vision Research, 28, 355-359.

Yeh, T. J., Pokorny, J., \& Smith, V. C. (1993). Chromatic discrimination with variation in chromaticity and luminance: Data and theory. Vision Research, 33, 1835-1845.

(Manuscript received February 8, 2000; revision accepted for publication February 22, 2001.) 